\title{
Correction: Macrophage-derived CCL5 facilitates immune escape of colorectal cancer cells via the p65/STAT3-CSN5-PD-L1 pathway
}

Chao Liu - Zhaoying Yao · Jianing Wang - Wen Zhang • Yan Yang • Yan Zhang • Xinliang Qu • Yubing Zhu • Jianjun Zou • Sishi Peng • Yan Zhao • Shuli Zhao • Bangshun He - Qiongyu Mi • Xiuting Liu • Xu Zhang • Qianming Du iD

Published online: 5 February 2020

(c) The Author(s), under exclusive licence to ADMC Associazione Differenziamento e Morte Cellulare 2020

\section{Correction to: Cell Death \& Differentiation}

https://doi.org/10.1038/s41418-019-0460-0

The original PDF version of this Article incorrectly showed two affiliations for corresponding author Qianming Du.
The authors asked for affiliation 2 (China Pharmaceutical University) to be removed, leaving only 7 (General Clinical Research Center, Nanjing First Hospital, Nanjing Medical University, Nanjing 210006, PR China).

This has been corrected in the PDF and HTML versions. 